\title{
The ecopsychosocial provision for psychiatric environments
}

\section{Evangelia Chrysikou}

Environment affects quality of care. Yet there are very few evaluation methods to address such issues. The SCP model is a user-inclusive, multi-paramentric method for the planning, design and evaluation of psychiatric facilities. Its application to psychiatric wards indicated hiatus between top-down (designers, building commissioning authorities) vs bottom up (patients and staff) understanding of what constitutes a therapeutic environment. That generated the need to investigate the socio-spatial context of psychiatric wards. This research aimed to promote our understanding of psychiatric space in relation to social interaction. Two acute wards in the UK provided the locus. Each was evaluated using the SCP model institutional vs domestic checklist, open-ended interviews with 10 staff and 12 patients and auditing of the spaces. Then, a Space Syntax analysis using Depthmap was employed to model social activity inside the wards. The SCP model analysis indicated that the wards, one of them new and purpose built and the other part of an old psychiatric campus soon to be replaced, demonstrated strong institutional features, even compared to previous samples using the same tools. The juxtaposition with space syntax demonstrated that areas mapped as integrated, i.e., socio-friendly, were areas where antisocial behaviours were observed. This could be interpreted by Goffman's theory on total institutions, as institutional spaces might be the context of reverse socio-spatial norms according to Hillier's social logic of space. Thus, institutional spaces could still house mental health provision. Integrated approaches for evaluating healthcare facilities for fit for purpose and social integration need to become part of a healthcare evaluation system. Healthcare provision should be evaluated for ecopsychosocially supportive environments.

Key message 1: The project raised the question of the appropriateness of mental healthcare environments

Key message 2: The spaces for the treatment and care of psychiatric patients might not be fit for their social re-integration 